\title{
LAS ACCIONES DIDÁCTICAS DEL PROFESORADO NOVEL UNIVERSITARIO: UN ESTUDIO CUALITATIVO DESDE UNA PERSPECTIVA INTERDISCIPLINAR
}

\author{
Marcos Jesús Iglesias Martínezㄹ, Inés Lozano Cabezas² ${ }^{2}$ Martha Sarmiento Osorio³ \\ Resumen
}

Las reformas europeas universitarias han transformado las maneras de enseñar y aprender en la Educación Superior. Las voces de los docentes sobre su práctica educativa relevan qué modelos educativos desarrollan en sus aulas y evidencian la efectividad de las reformas políticas. Este artículo investiga, desde una perspectiva interdisciplinar, sobre cómo enseñan los docentes noveles universitarios. Se adopta un enfoque cualitativo que analiza reflexiones de los participantes a través del soporte informático AQUAD.

Los resultados manifiestan que el profesorado novel aboga por un modelo centrando en los contenidos teóricos. Sin embargo, se identifican diferencias entorno a los métodos docentes y el sistema de evaluación en función del área de conocimiento. Las principales conclusiones evidencian la importancia de continuar indagando acerca del pensamiento de los noveles universitarios, pues son la piedra angular de las nuevas generaciones y sus reflexiones contribuyen a sistematizar los programas de formación pedagógica del profesorado novel en la Educación Superior.

Palabras clave: profesorado novel universitario, educación superior, desarrollo profesional, investigación cualitativa

\section{THE PRACTICES OF THE NOVICE LECTURERS: AN INTERDISCIPLINARY QUALITATIVE STUDY}

\begin{abstract}
European university reforms have transformed the ways of teaching and learning in Higher Education. Teachers' voices about their educational practice reveal what educational models they develop in their classrooms and demonstrate the effectiveness of political reforms. This article investigates, from an interdisciplinary perspective, on how new university teachers teach.

A qualitative approach is adopted that analyzes the reflections of the participants through the AQUAD computer support. The results show that the new teaching staff advocates a model focusing on theoretical content. However, differences are identified around teaching methods and the evaluation system depending on the area of knowledge. The main conclusions show the importance of continuing to inquire about the thinking of the new university students, since they are the cornerstone of the new generations and their reflections help to systematize the pedagogical training programs for new teachers in Higher Education.
\end{abstract}

Keywords: novice lecturers, higher education, professional development, qualitative research

Historial del artículo:

Fecha de recibido: 20-03-2020 - Fecha de aceptado: 28-05-2020

(C)2020 Fundación Universitaria Juan N. Corpas. FUJNC.

Artículo Open Access bajo la Licencia Creative Commons Atribución-NoComercial-Compartirlgual 4.0 Internacional. DOI: 10.26752/revistaparadigmassh.v1i2.468.

1. Doctor en Pedagogía con premio extraordinario. Docente y secretario académico del Departamento de Didáctica General y Didácticas Específicas de la Facultad de Educación en la Universidad de Alicante, España. Autor para correspondencia. Correo electrónico: marcos.iglesias@ua.es

2. Doctora en Pedagogía con premio extraordinario. Docente y Vicedecana de la Facultad de Educación en la Universidad de Alicante, España. Correo electrónico: ines.lozano@gcloud.ua.es

3. Maestrante en Educación para la Salud. Coordinadora Académica de la Escuela de Educación y Ciencias Sociales de la Fundación Universitaria Juan N. Corpas, Bogotá, D.C., Colombia. Correo electrónico: martha.sarmiento@juanncorpas.edu.co 


\section{Introducción}

Las tensiones y distensiones derivadas de la evolución de la sociedad del conocimiento o de la información (Castells, 2000) provocan alteraciones en las instituciones tradicionales de la Educación Superior del mundo occidental. A principios de siglo la Unión Europea en materia de Educación Superior (Broucker, De Wit, Verhoeven y Leisyte, 2019) asumió un momento de transformación radical provocado por las alteraciones sociales (Beck, 2000, 2004), a lo que la universidad, como transmisora y generadora de conocimiento, tiene la responsabilidad social y global de responder a las demandas y a las exigencias de la nueva de los nuevos retos sociales y culturales. En estos últimos 20 años, las universidades europeas han modificado sus procesos educativos para dar apertura de nuevas oportunidades de aprendizaje en la formación de los estudiantes universitarios (Curaj, Deca y Pricopie, 2018).

El objetivo principal de la convergencia europea es asegurar que todos los estudiantes tengan acceso a una enseñanza y aprendizaje de calidad, y que se respondan a las demandas de la sociedad con la preparación de profesionales competitivos y eficaces (Wagenaar, 2019). Inequívocamente, el intenso esfuerzo que están desarrollando todas las universidades europeas exige un cambio estructural y sustancial en los sistemas universitarios europeos; así la estructura de las universidades y la metodología docente universitaria han sufrido modificaciones profundas para responder, en cierta medida, a una formación universitaria basada en la adquisición de competencias profesionales que se correspondan con las habilidades de la sociedad del conocimiento. Para ello es indispensable que la nueva situación afecte al pensamiento de los profesores, a sus prácticas en el aula y a los aprendizajes del alumnado.

Este cambio de paradigma supone una alteración radical en el modelo de enseñanza universitaria, porque el modelo de universidad europea centra toda su organización universitaria en la asunción de que el alumnado debe ser un actor activo y los docentes investigadores críticos de su acción en el aula (Korthagen y Nuijten, 2017). El logro de un adecuado desarrollo del conocimiento y una excelente adaptación a los cambios tecnológicos demandan un adecuado nivel de competencia y responsabilidad del capital humano (Hargreaves y Fullan, 2012; Fullan, 2016).

El propósito de este artículo es analizar y valorar las tendencias y procesos en las metodologías docentes propuestos en el proceso de Bolonia, lo que implica tener en cuenta la formación inicial del profesorado universitario (Amundsen, y Wilson, 2012), aspecto olvidado en las universidades españolas, puesto que ha dado más peso a la investigación que la docencia (Caballero y Bolívar, 2015).

\section{Los retos para el profesorado novel universitario}

Actualmente, podemos hacer una aproximación a la naturaleza del modelo educativo en la Educación Universitaria Europea, un modelo que, sin duda, exige un nuevo perfil profesional de la enseñanza. Valcárcel (2003) afirma que actualmente no existen ni se exigen unos sistemas 
institucionales para la formación pedagógica, tanto inicial como de perfeccionamiento para el docente universitario. En este sentido, para conseguir este objetivo es preciso una profesionalización del profesorado universitario, es decir, es necesario una formación pedagógica institucional y sistemática (Zabalza, Cid y Trillo, 2014), para que los docentes adquieran las competencias necesarias y sean capaces de contribuir a una enseñanza universitaria de calidad (Zabalza, 2012).

La renovación conceptual y metodológica que ha asumido la institución universitaria europea adquiere necesariamente una visión de la educación como un proceso individual de constitución, adquisición y transmisión del conocimiento y, a la vez, una idea de aprendizaje como proceso de mejora de la capacidad de participar en una comunidad de práctica o de discurso (Wenger, McDermott \& Snyder, 2002). La investigación y el aprendizaje compartidos hacen a la institución más efectiva, coordinada y coherente (Rué, 2015).

Asimismo, el aprendizaje de los docente (Kennedy, 2014) y el cambio de metodología debe apoyarse en alguno de los aspectos de su práctica profesional (Billett, Harteis y Gruber, 2014), a través de la implementación de innovaciones metodológicas en sus aulas (Klieger y Yakobovitch, 2012) creando redes de profesores que fomenten la discusión y el dialogo compartido (Lave y Wenger, 1991; Cochran-Smith y Lytle, 2009; Wenger, McDermott \& Snyder, 2002), para dar respuesta a las principales dimensiones del diseño de los aprendizajes desde una postura crítica: cómo conocer, cómo actuar, con qué procedimientos y cómo ser. Ello supone, por tanto, un cambio de metodología docente y de estrategias de aprendizaje. Son cambios de modelos no vivenciados previamente por el profesorado novel universitario, pues se transita desde la canónica lección magistral hasta cualquier trabajo de aula o laboratorio con presencia del docente, y con mayor o menor participación del alumnado.

Para Pedró (2005), la cuestión didáctica universitaria no ha sido nunca objeto de preocupación, no obstante, últimamente viene desafiada por dos fenómenos coincidentes. Por un lado, la masificación de las aulas en las universidades, y, por otro lado, la presión a favor de una mayor eficacia en términos de calidad del producto. Nos encontramos en este escenario definido por nuevas ideas sobre educación y nuevas herramientas al servicio de conocimiento, que exigen cambiar de metodología docente en las aulas, siendo los profesores las personas con mayor capacidad, competencia y valía para hacer realidad esta reforma común europea. En el un proceso de enseñanza y aprendizaje donde los contenidos se subordinan a las competencias a desarrollar y donde la pregunta crucial para el docente novel ya no es "¿qué explicaré hoy?", sino más bien, "¿qué actividades deben desarrollar los estudiantes?"

Actualmente, la práctica docente del novel universitario debe adaptarse a la incorporación de nuevas metodologías docentes alternativas: más participativas y más colaborativas (Huber, 2008) y un buen profesor universitario habrá de reunir estas competencias (Bain, 2006). Para dar respuesta a los problemas actuales resulta insuficiente la modificación de la metodología docente en unas cuantas aulas, 
objetivo que siempre han intentado los profesores. Durante el período dedicado a la planificación de un programa formativo es necesario reorganizar los distintos elementos metodológicos que configuran el proceso enseñanza-aprendizaje, de tal forma que nos permita alcanzar las competencias que se establezcan como aprendizajes adquiridos por los estudiantes que cursan una materia. Se hace necesario, por tanto, que los profesores noveles universitarios combinen las acciones didácticas con clases presenciales, con el trabajo sobre papel y con el estudio en el espacio virtual. Se trata, por tanto, de una nueva competencia del docente universitario (Cabero-Almenara y Palacios-Rodríguez, 2020; Prendes y Gutiérrez, 2013) capaz de utilizar los materiales didácticos pedagógicos combinados con el uso de las herramientas digitales, además de facilitar el aprendizaje de los estudiantes universitarios (Iglesias, Lozano y Martínez, 2013).

\section{Diseño y metodológica de la investigación}

Se opta por un enfoque cualitativo holístico (Hadfield y Chapman, 2016) como método que permite una possible evidence-based for teacher education (Cochran-Smith y Zeichner, 2005), siendo el objeto de estudio las narrativas (Bolívar y Domingo, 2019; Goodson, Antikainen, Sikes y Andrews, 2017) de los docentes noveles universitarios sobre aprendizaje profesional en los inicios en la práctica educativa en la Educación Superior. Para esta investigación se plantean tres cuestiones de investigación:

1) ¿Cuáles son las concepciones que tienen el profesorado novel universitario sobre su práctica educativa?
2) ¿Qué características o atributos se identifican en los modelos de los profesores universitarios?

3) ¿Qué implicaciones se derivan para el diseño de los programas formativos para el profesorado novel universitario?

\section{Participantes}

En esta investigación participan de manera voluntaria 30 profesoras noveles y 30 profesores noveles pertenecientes a una Universidad pública española. La mayoría de los participantes tiene una edad entre de 26 y 35 años y pertenecen a las siguientes ramas de conocimiento: Ciencias Experimentales (25.51), Ciencias de la Salud (11.16\%), Ciencias Sociales y Jurídicas (31.67\%), Enseñanzas Técnicas (15\%) y Rama de Humanidades (16.67\%).

Para la presentación de esta investigación hemos considerado agrupar las áreas en dos cohortes denominadas área científica-técnica (a la cual pertenece las ramas de Ciencias Experimentales, de Ciencias de la Salud y de Enseñanzas Técnicas) y área social-humana (agrupando las ramas de Ciencias Sociales y Jurídicas y de Humanidades).

\section{Recogida y tratamiento de la información}

Se diseña una entrevista abierta semiestructurada validada (Onwuegbuzie y Leech, 2007) por cuatro expertos en investigación cualitativa y pertenecientes al área de Didáctica y Organización Escolar. Las preguntas de la entrevista invitaban a reflexionar a los docentes noveles sobre los aspectos relacionados con su acción 
didáctica en las aulas universitarias como son: la planificación didáctica, la metodología y el sistema de evaluación. Todas las entrevistas obtenidas se realizaron en formato audio, la cuáles fueron transcritas formato escrito.

Se utiliza el programa informático AQUAD 7 (Huber y Gürtler, 2012), el cual ha permitido conjugar en el proceso de interpretación y de codificación la interacción entre la emergencia de temáticas derivadas de las voces de los jóvenes profesores. El proceso de codificación se valida por dos expertos en investigación educativa, lo cual favorece una comprensión más profunda del fenómeno de estudio (Corbin y Strauss, 2008).

\section{Resultados}

En el análisis descriptivo y comparativo de este apartado se ha analizado y comparado los códigos referidos a la dimensión didácticopedagógica en relación a las distintas áreas de conocimiento a las que pertenecen los participantes. La identificación de las unidades semánticas de significado ha dado emergencia a cinco temáticas con sus correspondientes códigos. Los resultados se presentan en tablas para cada código, en las cuales se muestra el porcentaje de la frecuencia absoluta (\%FA) hallado para cada uno.

\section{Temática 1. Programación de la materia}

Esta temática emergente hace referencia a la preparación o planificación de los profesores sobre sus clases, a saber, la manera en la que los docentes planifican, diseñan, preparan o programan sus clases. En la tabla 1 se muestran los resultados referidos al profesorado novel del área científico-técnica.

\begin{tabular}{|l|c|}
\hline \multicolumn{2}{|l|}{ Tabla 1. La Programación Didáctica (área científico-técnica) } \\
\hline Código 1. Programación & $\% \mathrm{FA}$ \\
\hline 1.1. Programación de contenidos & $58.97 \%$ \\
\hline 1.2. Programación basada en el alumnado & $2.57 \%$ \\
\hline 1.3. Programación basada la coordinación docente & $38.46 \%$ \\
\hline TOTAL & $100 \%$ \\
\hline
\end{tabular}

Los resultados referidos al área científicotécnico destacan, principalmente, que la programación de los contenidos (código 1.1) predomina en las frecuencias analizadas en las narrativas de los participantes:

Resulta más sencillo realizaruna programación de los contenidos teóricos y prácticos, pues supone algo lineal en un curso académico. (Docen018).

Lo más importante es saber que deben saber los estudiantes. Si estamos una carrera de ciencias es muy importante que estos contenidos estén consolidados desde la base, por eso programo en contenidos. (Docen041)

Observamos, por otra parte, la importancia que se destaca en esta área de realizar una programación coordinada y colaborativamente entres varios colegas del área o del departamento y que, naturalmente, al igual que en el código 1.3 programan en base al aprendizaje del estudiante:

La programación de la asignatura está toda ya programada, porque yo voy a la par del profesor titular... entonces trabajamos un poco en equipo. (Docen020)

Nos reunimos con la coordinadora de la asignatura para organizar y decidir qué contenidos vamos a desarrollar. También esta coordinación nos permite seleccionar 
los materiales o recursos, y la verdad que cada año innovamos. (Docen059)

A continuación, en la tabla 2 se muestran los resultados referidos al profesorado novel del área social-humana.

Tabla 2. La Programación Didáctica (área social-humana)

\begin{tabular}{|l|l|}
\hline Código 1. Concepciones & \%FA \\
\hline
\end{tabular}

\begin{tabular}{|l|l}
\hline 1.2. Programación basada en el alumnado & $7.69 \%$ \\
\hline
\end{tabular}

\begin{tabular}{|l|l|}
\hline 1.3. Programación basada la coordinación docente & $3.85 \%$ \\
\hline
\end{tabular}

\begin{tabular}{|l|l}
\hline TOTAL & $3.85 \%$ \\
\hline
\end{tabular}

La programación de contenidos (código 1.1) presenta la frecuencia con más relatos. En este aspecto se observa que el profesorado novel de esta área considera más importante realizar una programación enfocada a los contenidos a trasmitir. Las dimensiones de esta planificación didáctica son en base a los objetivos, las competencias, los contenidos y el sistema de evaluación, tal y como se exponen en las siguientes narrativas.

Cuando comencé lo primero que me dijeron fue "Guía Docente" y que no podría salir de las competencias generales de la titulación y de la asignatura. De ahí que mis programaciones contengan todos los apartados de la Guía Docente. (DocenO28)

En los cursos del ICE siempre nos informaron de la importancia de programar sobre competencias, es decir, aquello que deben saber el alumnado. (Docen034)

A diferencia del área científico-técnico hallamos algunos relatos los cuales el profesorado novel manifiesten abiertamente que programan básicamente en función del aprendizaje del alumno (código 1.2). Una evidencia de ello el siguiente fragmento:
Normalmente lo cojo a través de los libros y yo voy viendo que si es la asignatura de [...], yo intento ver lo que se está dando o es lo que se ha introducido en estos libros. Quito muchas cosas que veo... por lo menos así ellos se sienten menos espectadores y por los menos participan más en las clases. (Docente).

\section{Temática 2. El tratamiento de los contenidos teóricos y prácticos}

Tabla 3. Tratamiento de los Contenidos de la Materia (área científico-técnica)

\begin{tabular}{|l|c|}
\hline Código 1. Contenidos de la materia & $\% \mathrm{FA}$ \\
\hline 2.1. Teóricos diferenciados de los prácticos & $72.22 \%$ \\
\hline 2.2. Vinculación teórico-práctico & $19.44 \%$ \\
\hline 2.3. Vinculación con las competencias profesionales & $8.34 \%$ \\
\hline TOTAL & $100 \%$ \\
\hline
\end{tabular}

En esta temática se agrupan las consideraciones de los participantes sobre las prácticas organizativas, en particular, en la articulación de los contenidos teóricos con los prácticos. En la tabla 3 se presentan los resultados obtenidos de los códigos emergidos en el área científico-técnica.

En el área científico-técnica se muestra un resultado más alto en el código 2.1, es decir, su cultura de organización de las actividades propende a separar los contenidos teóricos de los contenidos prácticos. Ello se debe, obviamente, que las prácticas suelen realizarse en los laboratorios y la teoría forma parte de las clases magistrales.

La metodología se ha basado en la distinción de clases teóricas y prácticas. (Docen012) Una cosa es práctica de laboratorio y otra cosa la teoría en el aula. Sí, se trata de manera 
diferenciada, no se puede realizar de otra manera. (Docen029)

Aunque parece de interés el que algunas narrativas se inclinen más por la conveniencia de relacionar la teoría y la práctica (código 2.2):

Por otra parte, cuando yo voy explicando en la teoría, pues suelo dividirlo en distintos bloques de 20 minutos, es decir, yo les suelo explicar la teoría y luego para saber si han entendido lo que yo les he explicado, pues les pongo un problema una actividad. Mi intención es ponerles lo problemas para motivarlos y así que aprendan lo que les estoy explicando y, también, para que lleven la cosas al día. (Docen018)

La verdad que no veo distinción, pues tanto teoría como las prácticas van unidas. (Docen050) El análisis del tratamiento de los contenidos del área social-humana se muestra en la tabla 4.

Tabla 4. Tratamiento de los contenidos de la materia (área social-humana)

\section{Código 1. Contenidos de la materia}

3.1. Teóricos diferenciados de los prácticos 3.2. Vinculación teórico-práctico

3.3. Vinculación con las competencias profesionales TOTAL

Observamos que mayoritariamente, el tratamiento de los contenidos por los profesores noveles del área social-humana está orientado a la práctica profesional de la titulación estudiada (código 2.3):

Digamos, vamos es que yo tiro más para la formación profesionalizante, aunque seamos de Filosofía y Letras esto del conocimiento no me termina a mí de cuajar porque aquí los cháveles terminan y que hacen después, entonces con el conocimiento. (Docen053) Formamos a maestros y eso hace que formemos a profesionales de la enseñanza. A mi lo que me interesa es enseñarles cosas relacionadas con su futura práctica profesional, es decir, lo que van utilizar o necesitar un futuro maestro. (Docen048)

También se considera relevante la importancia de vincular tanto contenidos teóricos como prácticos como una necesidad permanente, ya que el profesorado novel lo considera como inseparable (código 2.2):

No podría diferenciar entre teoría y práctica, siempre van unidos ¿no? (Docen009)

\section{Temática 3. Metodología docente implementada}

En esta temática hemos comparado qué metodología usan en cada de las áreas de conocimientos analizadas, es decir, la metodología que desarrollan los jóvenes profesores en sus aulas universitarias. En la tabla 5 hemos agrupado los códigos que han emergido en el área científico-técnica.

Tabla 5. Metodología Docente (área científico-técnica)

\begin{tabular}{|l|c|}
\hline Código 1. Metodología docente & \%FA \\
\hline 3.1. Basada en la enseñanza & $20.70 \%$ \\
\hline 3.2. Basada en el aprendizaje & $43.10 \%$ \\
\hline 3.3. Basada en la participación activa en la e-a & $36.20 \%$ \\
\hline TOTAL & $100 \%$ \\
\hline
\end{tabular}

Las narrativas del profesorado novel del área experimentales tienden a una metodología centrada en el alumnado, es decir, diseñar actividades y propuestas didácticas en el que el estudiante universitario sea el protagonista. En estos fragmentos manifiestan esta importancia recogida en el código 3.2.: 
Las clases teóricas son más variadas, desde trabajos en grupo y presentaciones en clase, celebración de debates, resolución de comentarios de cuadros, gráficos, análisis de textos, etc. (Docen012)

Pregunto, me preocupo, les insisto, les vuelvo a preguntar, no sé, pienso que es importante saber si me comprenden. (Docen040)

La participación en el proceso de enseñanzaaprendizaje (código 3.3) es descrito también comounaspectoenel diseño delametodología docente de los noveles en el grupo científico. La importancia de la interacción entre el alumnado y el profesorado es esencial para garantizar el éxito del proceso educativo en las aulas universitarias:

Yo no hago como hicieron conmigo, pienso que la intervención del alumnado es necesaria. (Docen31)

Hay que parar la clase para que participen, sobre todo en el laboratorio. (Docen009)

Los resultados de la segunda cohorte se muestran en la tabla 6.

Tabla 6. Metodología Docente (área social-humana)

\begin{tabular}{|l|c|}
\hline Código 1. Metodología docente & \%FA \\
\hline 3.1. Basada en la enseñanza & $16.00 \%$ \\
\hline 3.2. Basada en el aprendizaje & $34.00 \%$ \\
\hline 3.3. Basada en la participación activa en la e-a & $50.00 \%$ \\
\hline TOTAL & $100 \%$ \\
\hline
\end{tabular}

Las narrativas de los profesores noveles pertenecientes al área social-humana relatan que la metodología utilizada es participativa e interactiva con el alumnado, aspecto que hemos aglutinado con una alta frecuencia de aparición en el código 3.3: [D]as más participación al alumno y a mí me gusta generar debates, presentar materiales no únicamente... no sólo contar una historia, por eso me apoyo mucho en material visual, fotografías, etc. (Docen032)

Yo estoy mucho por grupos pequeños, de discusión, de mucha participación en clase, de mucha reflexión, no soy un profesor que me guste mucho, en el hecho de memorizar cosas o de dar contenidos o que los alumnos estén copiando en clase, prefiero que la gente piense que los alumnos piensen y que podamos discutir en clase y que hablen entre ellos que discutan entre ellos, pues creo que enriquece escuchar las opiniones de los demás alumnos y la opinión del profesor. (Docen005)

En coherencia con el código anterior, algunas narrativas también consideran la importancia del protagonismo del alumnado (código 3.2):

Entre ellos tienen que hacer lo mismo y lo hacen en grupos o individual, cada vez así. (Docen003)

[H] acemos ejercicios para que desarrollen los conocimientos que han adquirido, entonces, a mí me gusta más, más que magistral combinación de las dos. (Docen021)

\section{Temática 4. Utilización e integración de las telecomunicaciones digitales}

Los códigos emergidos en la temática 4 hacen referencia a la aplicación e integración de las tecnologías de la información y de la comunicación para el desarrollo de las clases, así como la utilización de plataformas digitales institucionales o de libre acceso en el proceso educativo en las aulas universitarias. 
Los resultados de ambas áreas se muestran en las tablas 7 y 8 respectivamente.

Tabla 7. Recursos Digitales (área científico-técnica)

\begin{tabular}{|l|c|}
\hline Código 1. Recursos digitales & \%FA \\
\hline 4.1. Integración en la enseñanza & $69.06 \%$ \\
\hline 4.2. Integración en el aprendizaje & $16.66 \%$ \\
\hline 4.3. Potencia y favorece la participación & $14.28 \%$ \\
\hline TOTAL & $100 \%$ \\
\hline
\end{tabular}

En el caso de la primera área, la integración de las telecomunicaciones digitales únicamente se utiliza de manera mayoritaria para facilitar los contenidos que el docente ha utilizado en el aula (código 4.1). Se aplica, pues, como un recurso más al servicio de la trasmisión de los contenidos, pero sin transformación pedagógica, sin modificaciones en la perspectiva ni en el procedimiento. Los segmentos de las narrativas referidos a esta categoría se refieren, exclusivamente, a la inclusión de presentaciones en PowerPoint, la publicación de documentos o de los temarios en el campus virtual, etc. Relatos referidos a este código son:

Si, yo creo que las utilizo bien. Todos los materiales y apuntes que utilizo se los publico. (Docen025)

Hago una selección de publicaciones científicas, las que utilizo para mis clases. Hoy en día está en la Web y las revistas suelen ser más accesibles. (Docen031)

El código 4.2 informa acerca la utilización de los recursos digitales para la mejora del aprendizaje de manera individual, a través de actividades, vídeos, demostraciones o el uso de plataformas digitales para un desarrollo óptimo del proceso de enseñanzaaprendizaje:
Suelo intercalar mis clases con los PowerPoint, pues con ello puedo introducir alguna que otra práctica, demostraciones, escribir preguntas con respuestas sencillas, motivadoras, actividades que sean Ilamativas (Docen017)

En la tabla 8 se presentan los resultados obtenidos en esta temática para el área social-humana.

Tabla 8. Recursos Digitales (área social-humana)

\begin{tabular}{|l|c|}
\hline Código 1. Recursos digitales & \%FA \\
\hline 4.1. Integración en la enseñanza & $54.28 \%$ \\
\hline 4.2. Integración en el aprendizaje & $17.15 \%$ \\
\hline 4.3. Potencia y favorece la participación & $28.57 \%$ \\
\hline TOTAL & $100 \%$ \\
\hline
\end{tabular}

Aún se manifiesta el uso de las telecomunicaciones digitales para presentar o compartir la información y los contenidos que emplea el docente novel en su práctica educativa (código 4.1):

Sí, el PowerPoint es el recurso básico. Además, te lo piden los alumnos que lo publique antes de comenzar las clases. (Docen014)

Yo preparo un documento en PDF y se publico en el campus virtual, para que no se pierda nadie de lo que vamos haciendo. (Docen044)

Los recursos digitales fomentan y favorecen la participación y colaboración del docente y del grupo clase empleando diferentes medios como son los debates virtuales, grupos discusión telemáticos, prácticas en grupo a través de plataformas digitales entre otros. Este aspecto ha sido también un código que ha emergido con alta frecuencia en el área social-humana. Un ejemplo del código 4.3 lo manifiesta la siguiente narrativa: 
Aver, algo, pues... todo eso sí que lo hacemos, pues lo de los debates virtuales, pero los grupos más pequeños en los que puedes ir tú guiándolos, ellos se involucran más, hay más interacción, etc. (Docen033)

\section{Temática 5. Sistema y procesos de evaluación}

Como síntesis final se han codificado los fragmentos referidos al sistema de evaluación que diseñan los docentes noveles, dando como resultado esta quinta temática cuyo análisis se refleja en las tablas 9 y 10.

\begin{tabular}{|c|c|}
\hline Código 1. Contenidos de la materia & \%FA \\
\hline 5.1. Sumativa (final) & $36.12 \%$ \\
\hline 5.2. Continua & $38.88 \%$ \\
\hline 5.3. Sumativa (final) y continua & $25.00 \%$ \\
\hline TOTAL & $100 \%$ \\
\hline
\end{tabular}

En el área más técnica se observa que no hay un consenso a la hora de desarrollar un sistema de evaluación, pues aún se mantiene la importancia de la evaluación sumativa:

Al final siempre vamos directos al examen final, es decir, le damos más valor a lo que cada uno sabe en esa prueba. (Docen049)

El sistema de evaluación siempre se ha dado preferencia al examen final, aunque también suman las prácticas. (Docen016)

También cabe destacar que los principios de Bolonia tienden más a una evaluación continua (código 5.2), en el que el profesorado novel es consciente de que la normativa es lo que exige y, por tanto, sus diseños evaluativos se basan en esas directrices:
Si diseñamos prácticas, habrá que corregirlas, y eso es lo que hacemos durante todo el cuatrimestre. En realidad es lo que vienen establecido en la Guía Docente, y también la normativa de la universidad. (Docen010)

El sistema de evaluación continua, no debemos salirnos de la norma. (Docen016)

Las evidencias en el área social-humana (tabla 10) manifiestan que sus procesos de evaluación van orientados a los principios establecidos en el Espacio Europeo de Educación Superior.

Tabla 10. Sistema de evaluación (área social-humana)

\begin{tabular}{|l|c|}
\hline Código 1. Contenidos de la materia & \%FA \\
\hline 5.1. Sumativa (final) & $27.04 \%$ \\
\hline 5.2. Continua & $54.05 \%$ \\
\hline 5.3. Sumativa (final) y continua & $18.91 \%$ \\
\hline TOTAL & $100 \%$ \\
\hline
\end{tabular}

La evaluación continua (código 5.2) es una de las máximas en el proceso de evaluación de esta área, en el que le dan importancia al desarrollo del proceso educativo. El profesorado novel de esta área manifiesta que es importante evaluar prácticas, pruebas y trabajos desarrollados por el alumnado.

En principio consideré que para evaluar los conocimientos en función de lo que habíamos visto lo mejor era hacer un examen, pero la evaluación continua permite evaluar todo el proceso. (Docen008)

La evaluación es continua, así lo hacemos desde la coordinación. No podemos hacer lo que cada uno nos guste, pues suponen agravios comparativos entre los diferentes grupos. (Docen007) 


\section{Discusión y conclusiones}

La valoración global comparativa entre las dos áreas de conocimiento muestra que la mayoría de relatos de los participantes tienden a diseñar un modelo de programación basado en los contenidos a impartir (Pill, 2005). Es importante señalar como excepción que en las áreas de enseñanzas técnicas el diseño de la programación se basa en la coordinación. Esta distintivita atesta una cultura de programación más coordinada en los estudios de las ingenierías y unas prácticas de programación más adaptadas sus estudios. En cualquier caso, deseamos manifestar que los estudiantes universitarios son los verdaderos protagonistas de su aprendizaje y la educación funciona como un ecosistema donde han de participar todos los implicados en su proceso (Dobber et al, 2013; Williams, 2014), aspecto no reflejado en los hallazgos de esta investigación.

Resulta asimismo interesante constatar que en las áreas científicas y técnicas (que tienden a ofrecer más salidas profesionales), los contenidos se organizan de manera separada diferenciando en dos bloques relativamente poco articulados entre sesiones teóricas y prácticas (Marcelo et al. 2014). Sin embargo, en el área social-humana el tratamiento de los contenidos se realiza mediante una combinación entre la teoría y la práctica o inclusive, articulando las sesiones con las actividades prácticas y con el estudio de la práctica profesional (Iglesias, Moncho y Lozano, 2019).

Esta diferencia tan acentuada puede deberse a que los profesores noveles pertenecientes a las ramas más técnicas o científicas, realizan obligatoriamente sus prácticas en lugares externos al aula de las sesiones teóricas (laboratorios, salidas de campo, prácticas externas, etc.) y este hecho obligue a diferenciar el contenido teórico del práctico. Inclusive, en la educación científica y técnica no es raro que distintos docentes se ocupen de cada uno de los dos dominios, teórica y práctica. Inequívocamente, las narrativas permiten afirmar que la articulación coherente entre teoría y práctica es uno de los ámbitos que conviene mejorar en la educación universitaria (Rodríguez y Santana, 2015).

Entre las prácticas educativas efectivas hay que adicionar los esfuerzos de los profesores noveles para incorporar estrategias metodológicas activas y participativas (Huber, 2008). Así, dos áreas relatan que las metodologías implementadas se centran en el aprendizaje de los estudiantes e intentan desarrollar una metodología participativa e innovadora, en la cual los estudiantes son protagonistas en esta metodología.

Se identifica una clara utilización de las telecomunicaciones digitales para la enseñanza (temática 5), de manera generalizada en las dos áreas comparadas (Cabero y Barroso, 2016; Mishra y Koehler, 2006). Entre las distintividades es significativo resaltar la aplicación de las herramientas digitales, singularmente, online, para fomentar un aprendizaje a través de la participación, sobre todo, en el área socialhumana. Posiblemente esta diferencia, con la otra área, se deba a que en área social-humana (más vinculada a los contextos lingüísticos) propicie más la posibilidad de fomentar debates o foros online participativos. Como contraste más significativo conviene apuntar que el área científico-técnica los participantes 
no han explicitado el uso social de los media como medio relevante en la programación y práctica educativa. Esta ausencia no es suficiente para afirmar que en dichos estudios técnicos no se apliquen redes tecnológicas o redes sociales.

En lo que se refiere a la evaluación, los resultados de la comparativa por áreas nos muestran que el área social-humanística utiliza, mayoritariamente, el sistema de evaluación continua (Parcerisa, 2014). Por otra parte, el área científico-técnico no se decanta por un sistema de evaluación en concreto - sumativa, continua o mixta -, puesto que hemos obtenidos resultados similares en los tres tipos de códigos referidos al sistema de evaluación (Reyes, Díaz Megolla, Pérez, Marchena y Sosa, 2020). Nuestra interpretación, en consonancia con los relatos, es que los jóvenes profesores han emprendido enormes esfuerzos para avanzar desde una evaluación sumativa gracias al estudio de la Didáctica, la cual ofrece una pluralidad de posibilidades.

Nuestras propuestas asumen la necesidad de una repensar la formación del profesorado novel universitario (Cochran-Smtih y Villegas, 2015), la constitución de la identidad profesional del profesorado novel ha de emerger desde las comunidades profesionales de práctica relacionadas en su contexto socio-cultural (Hadar y Brody, 2012). Este modelo permitir iniciar al profesorado novel universitario en su docencia y adaptarse a los nuevos escenarios educativos en la Universidad, y de este modo no reproducir los modelos docentes vivenciados en su formación académica, los cuales forman ya parte del pasado. Estamos convencidos que este aspecto puede jugar un gran cambio en el conocimiento de los docentes noveles, especialmente, si se sitúa en un aprendizaje entre colegas donde el trabajo colaborativo incita repensar críticamente sobre la práctica docente. La principal característica de los programas de desarrollo profesional docente novel debe situarse en la calidad de las experiencias de aprendizaje, ya que estos factores ayudan al joven profesor a formarse mediante la reflexión crítica y colectiva (Kezar, 2014) que les permite cuestionar su acción docente desarrolla en las universidades europeas.

\section{Referencias Bibliográficas}

1. Amundsen, C. \& Wilson, M. (2012). Are we asking the right questions? A conceptual review of the edu- cational development literature in higher education. Review of Educational Research, 82 (1), 90-126.

2.Bain, K. (2006). Lo que hacen los mejores profesores universitarios. Valencia: PUV.

3.Billett, S., Harteis, C. \& Gruber, H. (Editors) (2014). International Handbook of Research in Professional an Practice and Practice-based Learning. New York: Springer.

4.Broucker, B., De Wit, K., Verhoeven, J. C. \& Leisyte, L. (editors) (2019). Higher education system reform: An international comparison after twenty years of Bologna. Leiden: Brill Sense.

5.Bolívar, A. \& Domingo, J. (2019). La investigación (auto) biográfica en educación. Barcelona: Octaedro. 
6.Caballero, K. \& Bolívar, A. (2015). Identidades profesionales, concepciones de enseñanza-aprendizaje y estrategias docentes del profesorado universitario. Revista de Docencia Universitaria, 13 (1), 57-77.

7.Cabero-Almerana, J. \& Barroso, J. (2016). Formación del profesorado TIC: una vision del modelo TPACK. Cultura y Educación, 28 (3), 633-663.

8. Cabero-Almenara, J. \& Palacios-Rodríguez, A. (2020). Marco Europeo de Competencia Digital Docente "DiCompEdu" y cuestionario "Dig-CompEdu Check-In". EDMETIC, Revista de Educación Mediática y TIC, 9 (1), 213-234.

9. Castells, M. (2000). La era delainformación. Vol. I. Madrid: Alianza Editorial.

10. Cochran-Smtih, M. \& Lytle, S. L. (2009). Inquiry as Stance: Practitioner Research for the Next Generation. New York: Teachers College Press.

11. Cochran-Smtih, M. \& Villegas, A. M. (2015). Studying teacher preparation: the questions that drive research. European Educational Research Journal, 14(5), 379-394.

12. Cochran-Smith, M. \& Zeichner, K. M. (Eds.) (2005). Studying teacher education: The report of the AREA Panel on Research and Teacher Education. Mahwah, New Jersey: AERA-Lawrence Erlbaum Associates.
13. Corbin, J. \& Strauss, A. (2008). Basics of Qualitative Research (3rd ed.). Thousand Oaks, CA: SAGE.

14. Curaj, A. Deca, L. \& Pricope, R. (Editores) (2018). European Higher Education Area: the impact of past and future policies. Cham (Switzerland): Springer.

15. Dobber, M. et al. (2013). The development of community competence in the teacher education curriculum. European Journal of Teacher Education, 36 (3), 346-363.

16. Fullan, M. (2016). The NEW meaning of education change. Fith Edition. New York and London: Teachers College Press

17. Goodson, I., Antikainen, A. Sikes P. \& Andrews, M. (editors). (2017). The Routledge International Handbook on Narrative and Life History. New York: Routledge.

18. Hadar, L. L. \& Brody, D. L. (2012). The interaction between group processes and personal profes-sional trajectories in a professional development community for teacher educators. Journal of Teacher Education, 64(2), 45-61.

19. Hadfield, M. \& Chapman, C. (2016). Qualitative methods in educational improvement and effectiveness. En C. Chapman, D. Muiiss, D. Reynolds, P. Sammons \& C. Teddlie (eds.). The Routledge International Handbook of Educational Effectiveness and Improvement: Research, policy, and practice (pp. 202-219). New York: Routledge. 
20. Hargreaves, A. \& Fullan, M. (2012). Capital professional. Madrid: Ediciones Morata.

21. Huber, G. L. (2008). Aprendizajes activos y metodologías educativas. Revista de Educación, número extraordinario, 5981.

22. Huber, G. L. \& Gürtler, L. (2012). AQUAD 6. Manual del programa para analizar datos cualitativos. Tübingen: Ingeborg Huber Verlag.

23. Iglesias, M., Lozano, I. \& Martínez, M. (2013). La utilización de herramientas digitales en el desarrollo del aprendizaje colaborativo: análisis de una experiencia en Educación Superior. Revista Docencia Universitaria, 11(2), 333-351.

24. Iglesias, M., Moncho, M. \& Lozano, I. (2019). Repensando la formación teórica a través del Prácticum: experiencias de una docente novel. Contextos Educativos. Revista de Educación, 23, 49-64.

25. Kennedy, A. (2014). Understanding continuing professional development: the need for theory to impact on policy and practice. Professional Development in Education, 40(5), 688-697.

26. Kezar, A. (2014). Higher education change and social networks: A review of research. The Journal of Higher Education, 85 (1), 91-125.
27. Klieger, A., \& Yakobovitch, A. (2012). Contribution ofprofessional development to standards implementation. Teacher Development, 16(1), 77-88.

28. Korthagen, F.A.J. \& Nuijten, E.E. (2017). Core reflection approach in teacher education. En J. Lampert (Ed.), Oxford research encyclopedia of education. New York: Oxford University Press.

29. Lave, J. \& Wenger, E. (1991). Situated learning: Legitimate peripheral participation. New York: Cambridge University Press.

30. Marcelo García, C., Yot Domínguez, C., Mayor Ruiz, C., Sánchez Moreno, M., Murillo Estepa, P., Rodríguez López, J.M. \& Pardo Rojas, A. (2014). Las actividades de aprendizaje en la enseñanza universitaria: ¿hacia un aprendizaje autónomo de los alumnos?. Revista de Educación, 363, 334-359

31. Martín-Gutiérrez, Á., Conde-Jiménez, J. \& Mayor-Ruiz, C. (2014). La identidad profesional docente del profesorado novel universitario. Revista de Docencia Universitaria,12 (4). https://doi. org/10.4995/redu.2014.5618

32. Mishra, P. \& Koehler, M. (2006). Technological Pedagogical Content Knowlegde: a new framework for teacher knowledge. Teacher College Record, 108 (6), 1017-1054. 
33. Opfer, V. D., \& Pedder, D. (2011). Conceptualizing teacher professional learning. Review of Educational Research, 81(3), 376-407.

34. Onwuegbuzie, A. J. \& Leech, N. L. (2007). Validity and qualitative research: an oxymoron? Quality \& Quality: International Journal of Methodology, 41, 233-249.

35. Parcerisa, A. (coord.). (2014). Experiencias de evaluación continuada en la Universidad. Barcelona: Octaedro.

36. Pedró, F. (2005). Fauna académica. La profesión docente en las universidades europeas. Barcelona: Editorial UOC.

37. Pill, A. (2005). Models of profesional development in the education and practice of the new teachers in higher education. Teaching in Higher Education, 10 (2), 175-188.

38. Prendes, M. P. \& Gutiérrez, I. (2013). Competencias tecnológicas del profesorado en las universidades españolas. Revista de Educación, 361, 196-222.

39. Reyes García, C.I., Díaz Megolla, A., Pérez Solis, R., Marchena Gómez, R. \& Sosa Moreno, F. (2020). La evaluación del aprendizaje: percepciones y prácticas del profesorado universitario. Profesorado, revista de currículum y formación del profesorado, 24(1), 136-162. DOl: 10.30827/profesorado.v24i1.8449
40. Rodríguez, J. A. \& Santana, P. J. (2015). La distancia entre el trabajo del profesorado y del alumnado en el EEES: ¿Una brecha peligrosa? Profesorado, revista de currículum y formación del profesorado, 19(3), 380-404.

41. Rué, J. (2015). El desarrollo profesional docente en Educación Superior: agenda, referentes y propuestas para su adopción. REDU. Revista de Docencia Universitaria, 13, 217-236.

42. Valcárcel, M. (coord.) (2003). La preparación del profesorado universitario español para la convergencia europea en Educación Superior. Programa de estudios y análisis de la dirección general de universidades. Ministerio de Educación y Ciencia.

43. Wagenaar, R. (2019). REFORM! TUNING the Modernisation Process of Higher Education in Europe. A blueprint for student-centred learning. [Groningen]: University of Groningen.

44. Wenger, E. McDermott, R. A. \& Snyder, W. (2002). Cultivating communities of practice. Boston: Harward Bussiness Press.

45. Williams, J. (2014). Teacher Educator Professional Learning in the Third Space Implications for Identity and Practice. Journal of Teacher Education, 65(4), 315-326. 
46. Zabalza, M. A., Cid, A., \& Trillo, J. F. (2014). Formación docente del profesorado universitario. El difícil tránsito a los enfoques institucionales. Revista Española de Pedagogía, 257, 39-54.

47. Zabalza, M. A. (2012). El estudio de las "buenas prácticas" docentes en la enseñanza universitaria. Revista de Docencia Universitaria, 10 (1), 17-42. 